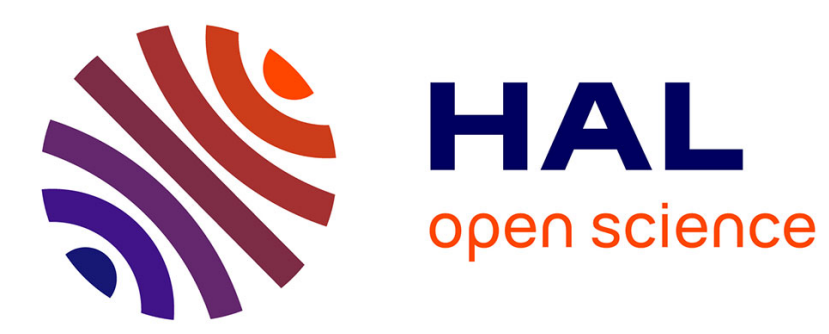

\title{
DNS of turbulent channel flow: can we imitate conjugate heat-transfer with a Robin boundary condition?
}

Cédric Flageul, Sofiane Benhamadouche, Eric Lamballais, Dominique

\author{
Laurence
}

\section{- To cite this version:}

Cédric Flageul, Sofiane Benhamadouche, Eric Lamballais, Dominique Laurence. DNS of turbulent channel flow: can we imitate conjugate heat-transfer with a Robin boundary condition?. 8th international symposium of Turbulence Heat and Mass Transfer , Sep 2015, Sarajevo, Bosnia and Herzegovina. hal-01323794

\section{HAL Id: hal-01323794 \\ https://hal.science/hal-01323794}

Submitted on 31 May 2016

HAL is a multi-disciplinary open access archive for the deposit and dissemination of scientific research documents, whether they are published or not. The documents may come from teaching and research institutions in France or abroad, or from public or private research centers.
L'archive ouverte pluridisciplinaire HAL, est destinée au dépôt et à la diffusion de documents scientifiques de niveau recherche, publiés ou non, émanant des établissements d'enseignement et de recherche français ou étrangers, des laboratoires publics ou privés. 


\title{
DNS of turbulent channel flow: can we imitate conjugate heat-transfer with a Robin boundary condition?
}

\author{
C. Flageul ${ }^{1}$, S. Benhamadouche ${ }^{1}$, E. Lamballais ${ }^{2}$ and D. Laurence La $^{1,3}$ \\ ${ }^{1}$ EDF R\&D, Fluid mechanics, Energy and Environment, 78401 Chatou, France, \\ cedric.flageul@edf.fr
}

${ }^{2}$ Institute PPRIME, Department of Fluid Flow, Heat Transfer and Combustion, Université de Poitiers, CNRS, ENSMA, Téléport 2 - Bd. Marie et Pierre Curie B.P. 30179, 86962 Futuroscope Chasseneuil Cedex, France

${ }^{3}$ School of Mechanical, Aerospace and Civil Engineering, The University of Manchester, Sackville Street, Manchester M60 1QD, UK

\begin{abstract}
Turbulent heat fluxes, temperature variance and associated budgets obtained from the Direct Numerical Simulation of an incompressible turbulent channel flow with a Reynolds number of 150 based on the wall friction velocity and a PrandtI number of 0.71 are presented and analysed for four different cases: imposed temperature at the wall (Dirichlet), imposed heat flux (Neumann), heat exchange coefficient (Robin) and 3D conjugate heat transfer. Present results show that a Robin boundary condition can imitate most of the one point statistics obtained with conjugate heat-transfer accurately compared to the statistics obtained with an imposed temperature or heat flux at the wall.
\end{abstract}

\section{Introduction}

Most of the DNS of the turbulent channel flow performed with a passive scalar are based on an imposed temperature at the wall (Kasagi et al. 1992, Kawamura et al. 1998). When the temperature is imposed at the wall, there is a close similarity between thermal and momentum low-speed near-wall streaks (Abe et al. 2009).

In a small number of DNS, a constant heat flux is imposed at the wall (Tiselj et al. 2001). However, it is widely recognized that neither isothermal nor isoflux boundary conditions can realistically mimic the actual heat transfer in real life where the wall has a thickness, especially when the thermal diffusivity of the fluid and solid are of the same order of magnitude. When such a coupling is considered, it is referred as conjugate heat-transfer. To the authors' knowledge Tiselj et al. (2001) were the first to investigate by DNS the influence of the thermal boundary conditions with a direct comparison between conjugate heat transfer, imposed temperature and imposed heat flux at the wall. 
In the present paper, the turbulent heat-fluxes, the temperature variance and the associated budgets obtained for three different boundary conditions (imposed temperature (iso T), imposed heat flux (isoQ), heat exchange coefficient (Robin)) and conjugate heat transfer (Conjug) are compared. In the present conjugate simulations, the ratio of thermal diffusivity and conductivity between the solid and the fluid can be equal to 2, 1 and 0.5: 9 conjugate simulations were performed (the heat flux is imposed at the outer wall of the solid domain).

\section{Numerical setup}

Present simulations are based on the open-source software Incompact3d (https://code.google.com/p/incompact3d/) developed at Université de Poitiers and Imperial College London by Laizet et al. (2011). The details of the numerical methods employed here are similar to the ones given in Flageul et al. (2014): high-order compact finite difference schemes in the fluid domain are combined with a mixed finite difference/spectral discretization in the solid domain. The number of computational cells is equal to $256 \times 193 \times 256$ and the domain size is $[25.6,2,8.52]$. For the conjugate heat transfer cases, the solid domain is on top and bottom of the fluid one, as sketched figure 1 . For such cases, the equation of evolution of the passive scalar reads

$$
\begin{gathered}
\partial_{t} T+\partial_{j}\left(T u_{j}\right)=\alpha_{f} \partial_{j j}^{2} T \text { in the fluid } \\
\partial_{t} T_{s}=\frac{\alpha_{f}}{G_{1}} \partial_{j j}^{2} T_{s} \text { in the solid } \\
T_{f}=T_{s} \text { and } \partial_{y} T_{f}=G_{2} \partial_{y} T_{s} \text { at the interface }
\end{gathered}
$$

where $G_{1}$ is the ratio of thermal diffusivities $\alpha_{f} / \alpha_{s}$ and $G_{2}$ is the ratio of thermal conductivities $\lambda_{s} / \lambda_{f}$. Based on those dimensionless numbers, the thermal activity ratio $K$ can be defined with $1 / K=G_{2} \sqrt{G_{1}}$ (Tiselj et al. (2012)). The ratio of thermal diffusivities and conductivities considered here lead to $K=\lfloor 1 / 2 \sqrt{2} ; 1 / 2 ; 1 / \sqrt{2} ; 1 ; \sqrt{2} ; 2 ; 2 \sqrt{2}\rfloor$. Both for $K=1 / \sqrt{2}$ and $K=\sqrt{2}$, two simulations were performed: $\left(G_{1}, G_{2}\right)=(2,1), \quad\left(G_{1}, G_{2}\right)=(1 / 2,2) \quad$ and $\left(G_{1}, G_{2}\right)=(1 / 2,1)$, $\left(G_{1}, G_{2}\right)=(2,1 / 2)$. Theoretical analysis shows that a very low (resp. high) thermal conductivity in the solid domain is equivalent to an imposed heat-flux (resp. imposed temperature) at the fluid boundary. Therefore, for a given $G_{1}$, the lower $G_{2}$, the higher $K$, the closer to the imposed heat flux case. Accordingly as illustrated in figure 1 for the temperature variance, the higher $K$, the closer "Conjugate" results move to the imposed heat flux case. 

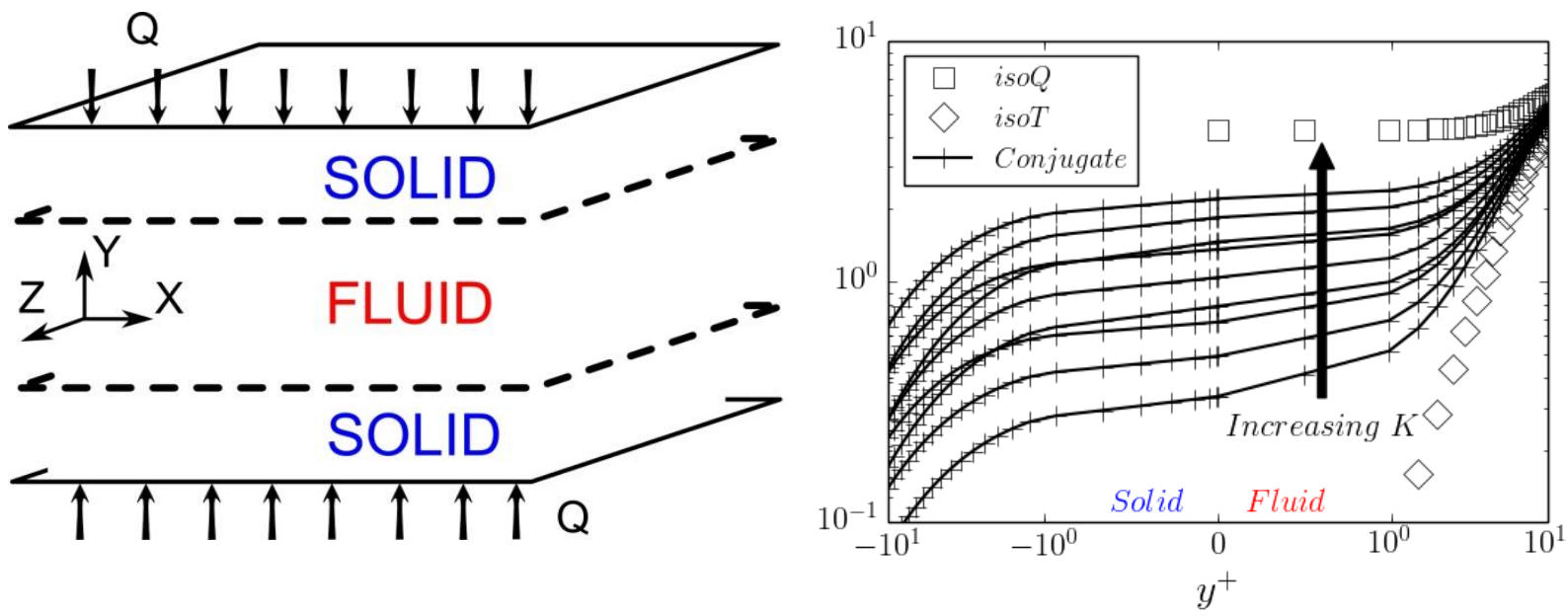

Figure 1: Left: Sketch of the configuration. Right: Temperature variance.

\section{Compatibility condition for the non-conjugate cases}

One considers a general boundary condition for the temperature at the wall $a T+b \partial_{y} T=c$. Furthermore, if one assumes the coefficients $a, b$ and $c$ are constant, one obtains:

$$
a T+b \partial_{y} T=c \Rightarrow a \bar{T}+b \partial_{y} \bar{T}=c \text { and } a T^{\prime}+b \partial_{y} T^{\prime}=0
$$

The second condition in (1) can be multiplied by $T^{\prime}$ or $\partial_{y} T^{\prime}$. The resulting 3 equations can be used to build a matrix:

$$
\left(\begin{array}{ccc}
\bar{T} & \partial_{y} \bar{T} & -1 \\
T^{\prime 2} & \partial_{y}\left(T^{\prime 2}\right) / 2 & 0 \\
\partial_{y}\left(T^{\prime 2}\right) / 2 & \partial_{y} T^{\prime} \partial_{y} T^{\prime} & 0
\end{array}\right)\left(\begin{array}{l}
a \\
b \\
c
\end{array}\right)=\left(\begin{array}{l}
0 \\
0 \\
0
\end{array}\right) \Rightarrow 4 T^{\prime 2} \times \partial_{y} T^{\prime} \partial_{y} T^{\prime}=\partial_{y}\left(T^{\prime 2}\right) \times \partial_{y}\left(T^{\prime 2}\right)
$$

The determinant of the matrix must vanish to allow non trivial solutions. The resulting relation connects the values (at the wall) of the temperature variance, its derivative and the wall-normal part of the dissipation rate associated with the temperature variance. One can combine the second and the third lines of the matrix in (3) to obtain: $a^{2} / b^{2}=T^{\prime 2} / \partial_{y} T^{\prime} \partial_{y} T^{\prime}$. Our Robin boundary condition for $\left(G_{1}, G_{2}\right)=(1,1)$ was built using this relation with the temperature variance from the iso $Q$ case and the wall-normal part of the dissipation rate from the isoT one. The Robin boundary condition for the other conjugate cases was obtained using cross-multiplication between the one at $\left(G_{1}, G_{2}\right)=(1,1)$, and the ratio $T^{\prime 2} / \partial_{y} T^{\prime} \partial_{y} T^{\prime}$ of the conjugate cases.

\section{Compatibility condition for the conjugate cases}

Assuming the solid domain is semi-infinite in the wall-normal direction and the 
case is stationary, one can apply Fourier and Laplace analysis to the solid heat diffusion equation:

$$
i k_{t} \frac{\rho C_{p}}{\lambda} T=\partial_{y y}^{2} T-\left[k_{x}^{2}+k_{z}^{2}\right] T \stackrel{\text { Laplace }}{\longrightarrow} T=\frac{s T_{y=0}+\partial_{y} T_{y=0}}{s^{2}-\left(k_{x}^{2}+k_{z}^{2}\right)-i k_{t} \frac{\rho C_{p}}{\lambda}}
$$

The denominator can be written as $s^{2}-S^{2}$ with $S^{2}=k_{x}^{2}+k_{z}^{2}+i k_{t} \rho C_{p} / \lambda$. Applying an inverse Laplace transform leads to a compatibility condition between the wall-normal heat flux and the temperature at the wall: $\partial_{y} T_{y=0} \pm S T_{y=0}=0$. The sign in the expression depends on the sign of the real part of $S$ and on the orientation of the y-axis. This condition is a product in the spectral space, which is equivalent to a convolution in physical space. If one were to neglect the dependency of $S$ on $k_{x}, k_{z}$ and $k_{t}$, the quadratic norm of the compatibility condition would be $1 / S^{2}=T^{\prime 2} / \partial_{y} T^{\prime} \partial_{y} T^{\prime}$. As this ratio is used to define our Robin boundary condition, there is strong connection between the conjugate cases and the Robin ones. Using a Robin boundary condition with constant coefficients, one can imitate conjugate heat transfer but neglect the non-local effects (i.e. the convolution).

\section{Results}

In figure 2, the temperature variance obtained for the conjugate cases and the Robin ones are in between the iso $T$ and the iso $Q$ one. There is a remarkable agreement between the conjugate cases and the Robin ones. It is similar for the dissipation rate associated with the temperature variance, although the agreement between the Robin case and the conjugate ones is degraded for $y^{+} \leq 7$. For the ratio of thermal properties considered in the present conjugate simulations, the temperature variance and the associated dissipation rate depends only on the thermal activity ratio $K$.

The one point correlation coefficient associated with the turbulent heat flux $<u_{j}^{\prime} T^{\prime}>$ is $\left\langle u_{j}^{\prime} T^{\prime}>/\left(u_{j R M S} T_{R M S}\right)\right.$. In figure 3 , the one point correlation coefficient associated with the wall-normal turbulent heat flux obtained with conjugate heat transfer and a Robin boundary condition lies in between the iso $T$ and isoQ cases. There is a good agreement between the Robin case and the conjugate ones, although the agreement is more qualitative for $y^{+} \leq 3$. The one point correlation coefficient associated with the streamwise turbulent heat-flux obtained with conjugate heat transfer and a Robin boundary condition lies in between the iso $T$ and iso $Q$ cases. The Robin cases are however now very close to the iso $T$ one. In addition, for a given thermal activity ratio, the one point correlation coefficient for the conjugate cases does not overlap (cases $K=1 / \sqrt{2}$ and $K=\sqrt{2}$ ). 
Therefore, the turbulent heat fluxes depends both on $K$ and $G_{1}$, even if the temperature variance and the associated dissipation rate depends only on $K$.

In figure 4, the two-point streamwise autocorrelation of the temperature at the wall for the Robin cases is lower than the conjugate and iso $Q$ ones. Oppositely, the two-point streamwise autocorrelation of the wall-normal heat flux at the wall for the Robin cases is higher than the conjugate and isoT ones. As the coefficients in the Robin boundary condition are constants, the autocorrelation of the temperature and wall-normal heat flux are identical. This is obviously not the case for conjugate heat transfer. This confirms the failure of a Robin boundary condition with constant coefficients to mimic the non-local aspects of conjugate heat transfer.

In figure 5, the two point spanwise autocorrelation of the temperature and wall-normal heat flux at the wall show a similar trend, although the impact of the thermal boundary condition is less severe.

In figure 2, the temperature variance in the fluid domain overlap for the simulations at $K=1 / \sqrt{2}$ and $K=\sqrt{2}$. As shown figure 6 , this is not the case in the solid domain. This leads to distinct second derivatives of the temperature variance in the solid domain (for a given thermal activity ratio $K$ ). In the budgets of the temperature variance in the solid domain, $\varepsilon_{\theta}$ balances the molecular diffusion. The small difference observed on $\theta^{2}$ in the solid domain leads to a larger difference on $\varepsilon_{\theta, s}$.

The relative contribution to $\varepsilon_{\theta}$ of its wall-normal part is $\partial_{y} T^{\prime} \partial_{y} T^{\prime} / \partial_{i} T^{\prime} \partial_{i} T^{\prime}$. As shown figure 7 (left), in the fluid domain, the wall-normal part of $\varepsilon_{\theta, f}$ is dominant in the near-wall region for the conjugate, Robin and iso $T$ cases. Therefore, in the vicinity of the wall, for the conjugate and Robin cases, the value of $\varepsilon_{\theta, f}$ is closer to the isoQ case while its anisotropy is closer to the isoT case. Using the continuity of temperature and heat-flux at the fluid-solid interface and the budgets of the temperature variance in the fluid and solid domains leads to the interface condition:

$$
2 \partial_{y} T_{f}^{\prime} \partial_{y} T_{f}^{\prime}\left(1-\frac{\lambda_{f}^{2}}{\lambda_{s}^{2}}\right)=\partial_{y y}\left(T_{f}^{\prime 2}\right)-\partial_{y y}\left(T_{s}^{\prime 2}\right)=\frac{\varepsilon_{\theta, f}}{\alpha_{f}}-\frac{\varepsilon_{\theta, s}}{\alpha_{s}} \Leftrightarrow \frac{\partial_{y} T_{f}^{\prime} \partial_{y} T_{f}^{\prime}}{\partial_{i} T_{f}^{\prime} \partial_{i} T_{f}^{\prime}}\left(1-\frac{1}{G_{2}^{2}}\right)=1-G_{1} \frac{\varepsilon_{\theta, s}}{\varepsilon_{\theta, f}}
$$

Assuming the wall-parallel contribution to $\varepsilon_{\theta, f}$ is negligible at the fluid-solid interface leads to:

$$
\frac{\varepsilon_{\theta, s}}{\varepsilon_{\theta, f}}=\frac{1}{G_{1} G_{2}^{2}}=K^{2}
$$

This is in good agreement with our results: the higher $K$, the higher $\varepsilon_{\theta, s} / \varepsilon_{\theta, f}$.

In figure 7 (right), the temperature variance in the solid domain near the 
fluid-solid interface seems to overlap when the abscissa and ordinate axes are rescaled with $G_{2}$ and $K$ respectively. However, the focus on the wall-value indicates large deviations.

The relative contribution of the wall-normal part of $\varepsilon_{\theta, f}$ at the wall as a function of $K$ seems linear with a slope of -0.19 for the ratio of thermal properties considered in this study. However, there are few chances for this trend to be valid for ratio of thermal properties farther from unity as this relative contribution is bounded in $[0,1]$.

\section{Discussion}

On the one hand, our results show that a Robin boundary condition can imitate accurately conjugate heat-transfer: the temperature variance and the associated budgets are in good agreement. On the other hand, the one point correlation coefficient associated with the streamwise turbulent heat flux and the streamwise autocorrelations at the wall show that there remains a difference between conjugate and non-conjugate heat-transfer.

From section 4 , in case of conjugate heat transfer, there is a compatibility condition that is a product in the spectral space, which is equivalent to a convolution in the physical space. Such a condition is non-local and cannot be imitated by a Robin boundary condition with constant coefficients. The theoretical failure of such a Robin boundary condition to mimic the non-local aspect of conjugate heat transfer is confirmed by our results.

The relatively high autocorrelation of the temperature at the wall for the conjugate cases at large separations may be explained by the periodicity and the limited streamwise extension of our domain, even if it is longer than the reference ones (Kasagi et al. 1992, Tiselj et al. 2001). Very large scale thermal structures may also be intrinsic to conjugate heat transfer: those are the only ones able to penetrate deep inside the solid domain. For instance, at $K=1 / \sqrt{2}$, the streamwise autocorrelation of the temperature at the wall at the largest separation is 0.19 for $\left(G_{1}, G_{2}\right)=(2,1)$ and 0.14 for $\left(G_{1}, G_{2}\right)=(1 / 2,2)$. The higher $G_{1}$, the lower the thermal diffusion in the solid domain, the more important are very large scale thermal structures.

\section{Conclusion}

From a RANS perspective, a Robin boundary condition with constant coefficients seems able to mimic conjugate heat transfer: the second moments and their budgets are satisfactorily reproduced (fig. 2). From a LES perspective, this is no longer the case, as shown by the streamwise autocorrelations of the temperature 
and wall-normal heat-flux at the wall (fig. 4).

Those conclusions may not be valid for ratio of thermal properties farther from unity than the ones considered here. When the Prandtl number is 0.01, the temperature variance at the wall depends both on $K$ and $G_{1}$ (Tiselj et al. (2012)).

Following the analysis developed in section 4, a Robin boundary condition with varying coefficient could offer a better potential to mimic conjugate heat-transfer. However, the coefficients should depend on values of the temperature and wall-normal heat flux at the wall in a non-local fashion: both previous time-steps and surrounding nodes should be considered to mimic the convolution accurately.

\section{Acknowledgements}

The authors thank the French National Research Agency (ANRT) and EDF R\&D for its contribution in funding the present study (CIFRE 2012/0047) and providing computational time on Zumbrota supercomputer (IBM - Blue-geneQ) respectively.

\section{References}

1. H. Abe, R. A. Antonia, and H. Kawamura. Correlation between small-scale velocity and scalar fluctuations in a turbulent channel flow. Journal of Fluid Mechanics, 627, 1-32, 2009.

2. C. Flageul, S. Benhamadouche, E. Lamballais, and D. Laurence. DNS of channel flow with conjugate heat transfer-Budgets of turbulent heat fluxes and temperature variance. In $10^{\text {th }}$ International ERCOFTAC Symposium on Engineering Turbulence Modelling and Measurements, 2014 (to appear in Int. J. of Heat and Fluid Flow).

3. N. Kasagi, Y. Tomita, and A. Kuroda. Direct numerical simulation of passive scalar field in a turbulent channel flow. Journal of heat transfer, 114(3), 598-606, 1992.

4. H. Kawamura, K. Ohsaka, H. Abe, and K. Yamamoto. DNS of turbulent heat transfer in channel flow with low to medium-high Prandtl number fluid. International Journal of Heat and Fluid Flow, 19(5), 482-491, 1998.

5. S. Laizet, and N. Li. Incompact3d: A powerful tool to tackle turbulence problems with up to $O\left(10^{5}\right)$ computational cores. International Journal for Numerical Methods in Fluids, 67(11), 1735-1757, 2011.

6. I. Tiselj, R. Bergant, B. Mavko, I. Bajsić, and G. Hetsroni. DNS of turbulent heat transfer in channel flow with heat conduction in the solid wall. Journal of heat transfer, 123(5), 849-857, 2001. 
7. I. Tiselj, and L. Cizelj. DNS of turbulent channel flow with conjugate heat transfer at Prandtl number 0.01. Nuclear Engineering and Design, 253, 153-160, 2012. 

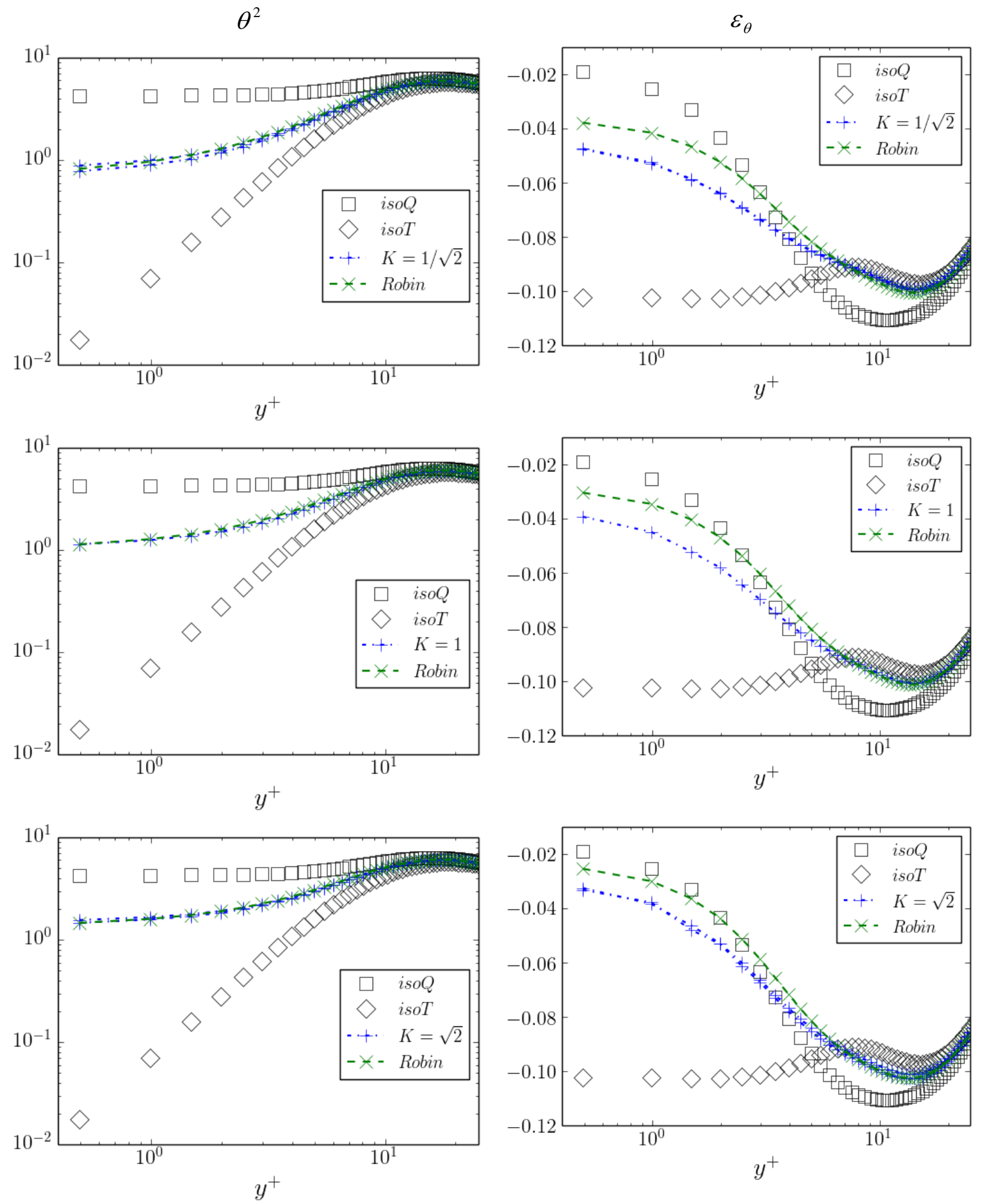

Figure 2. Left: Temperature variance. Right: Associated dissipation rate. Top: $K=1 / \sqrt{2}$. Middle: $K=1$. Bottom: $K=\sqrt{2}$. +: Conjugate. $x$ : Robin. 
Correlation (wall-normal turbulent heat

flux)
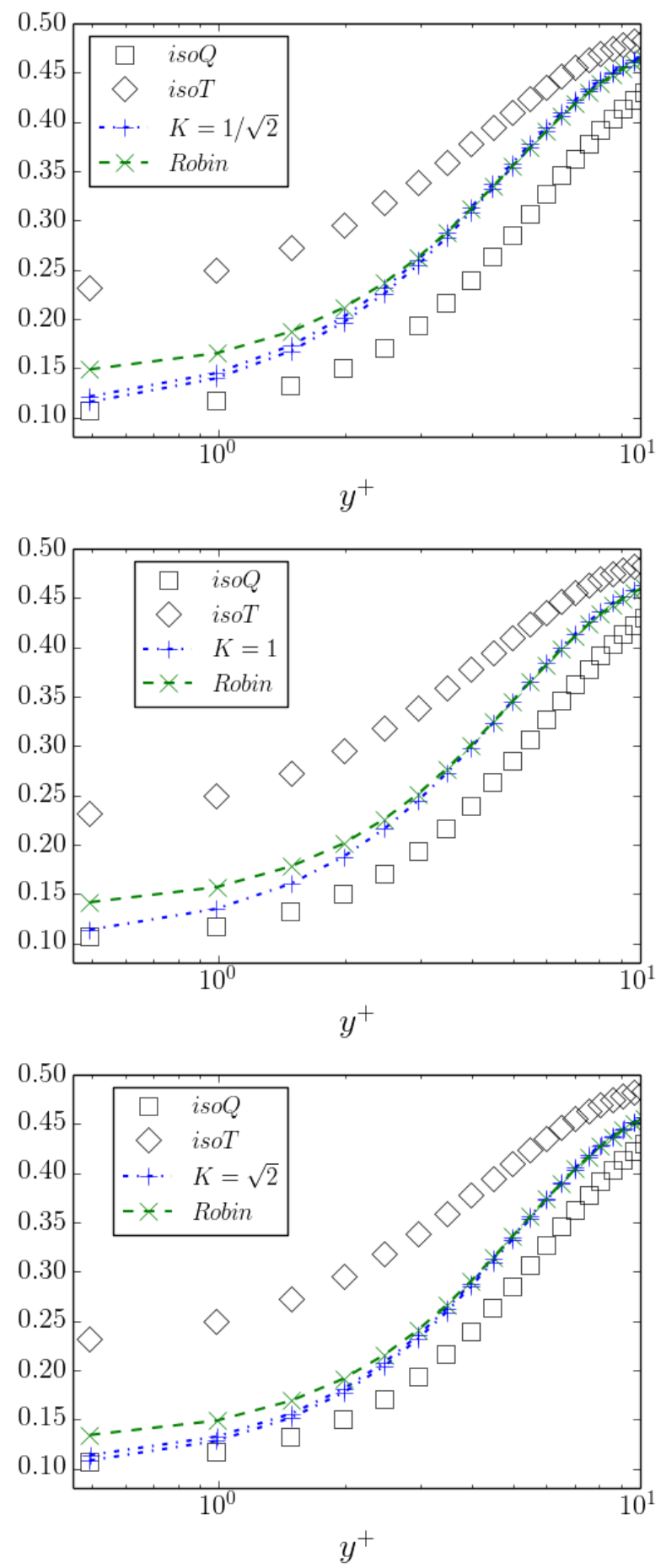

Correlation (streamwise turbulent heat flux)
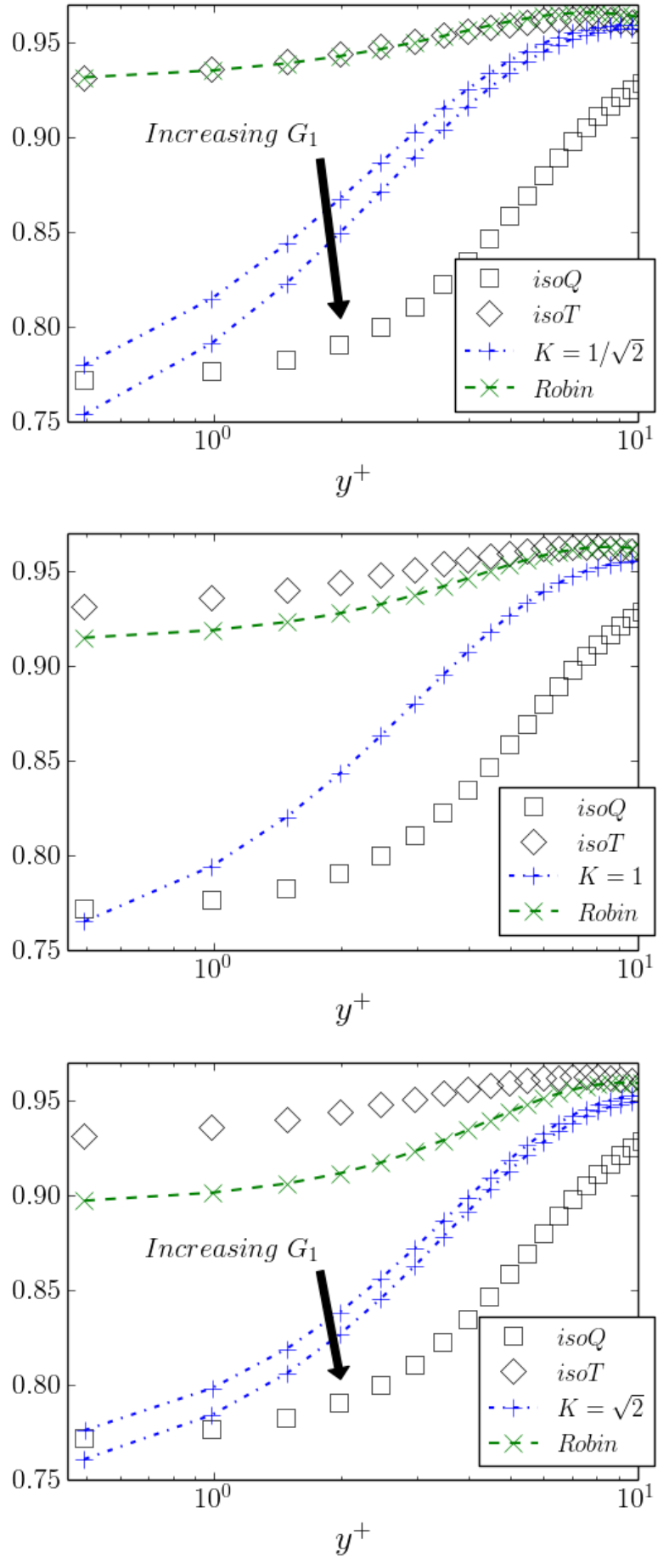

Figure 3: One point correlation coefficient associated with the turbulent heat flux. Left: Wall-normal turbulent heat flux. Right: Streamwise turbulent heat flux. Top: $K=1 / \sqrt{2}$. Middle: $K=1$. Bottom: $K=\sqrt{2}$. + : Conjugate. x: Robin. 
Two-point streamwise autocorrelation

(temperature)
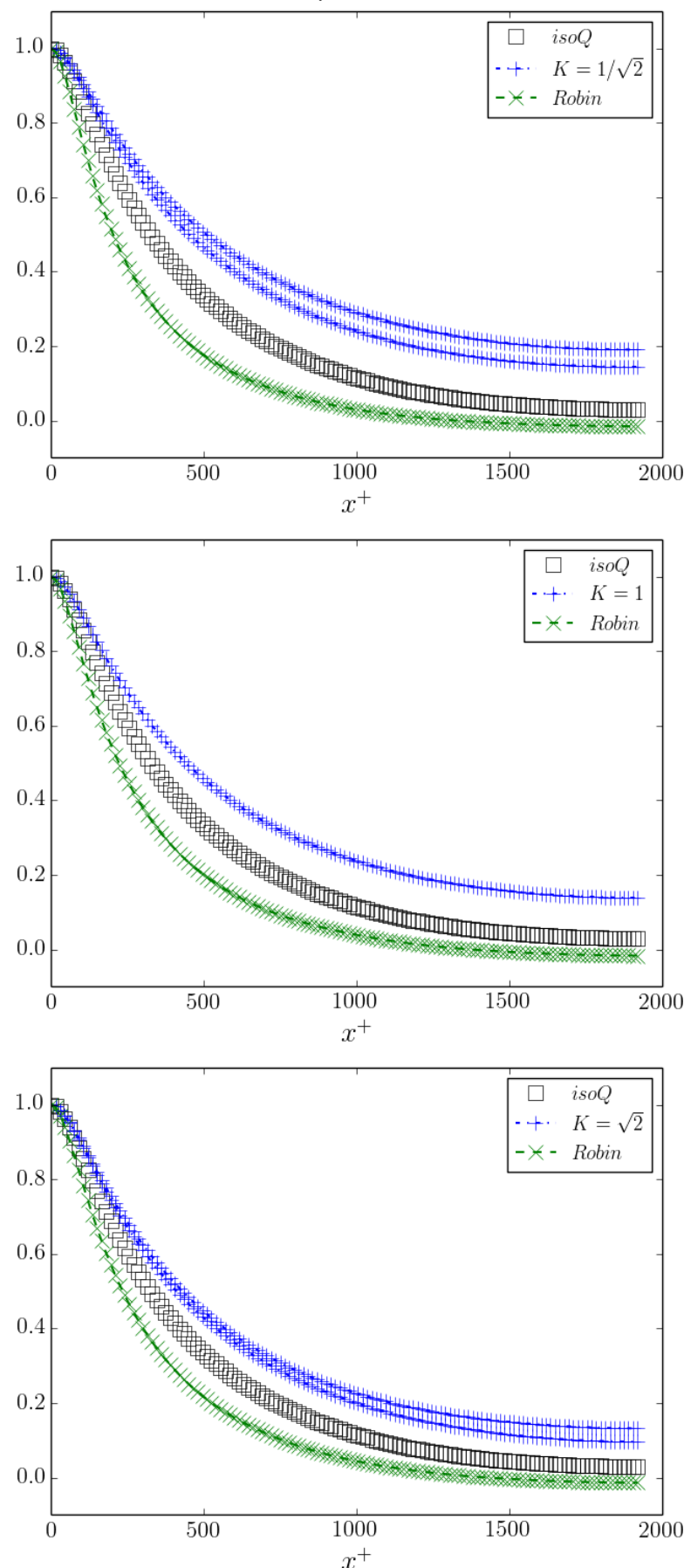

Two-point streamwise autocorrelation (wall-normal heat flux)
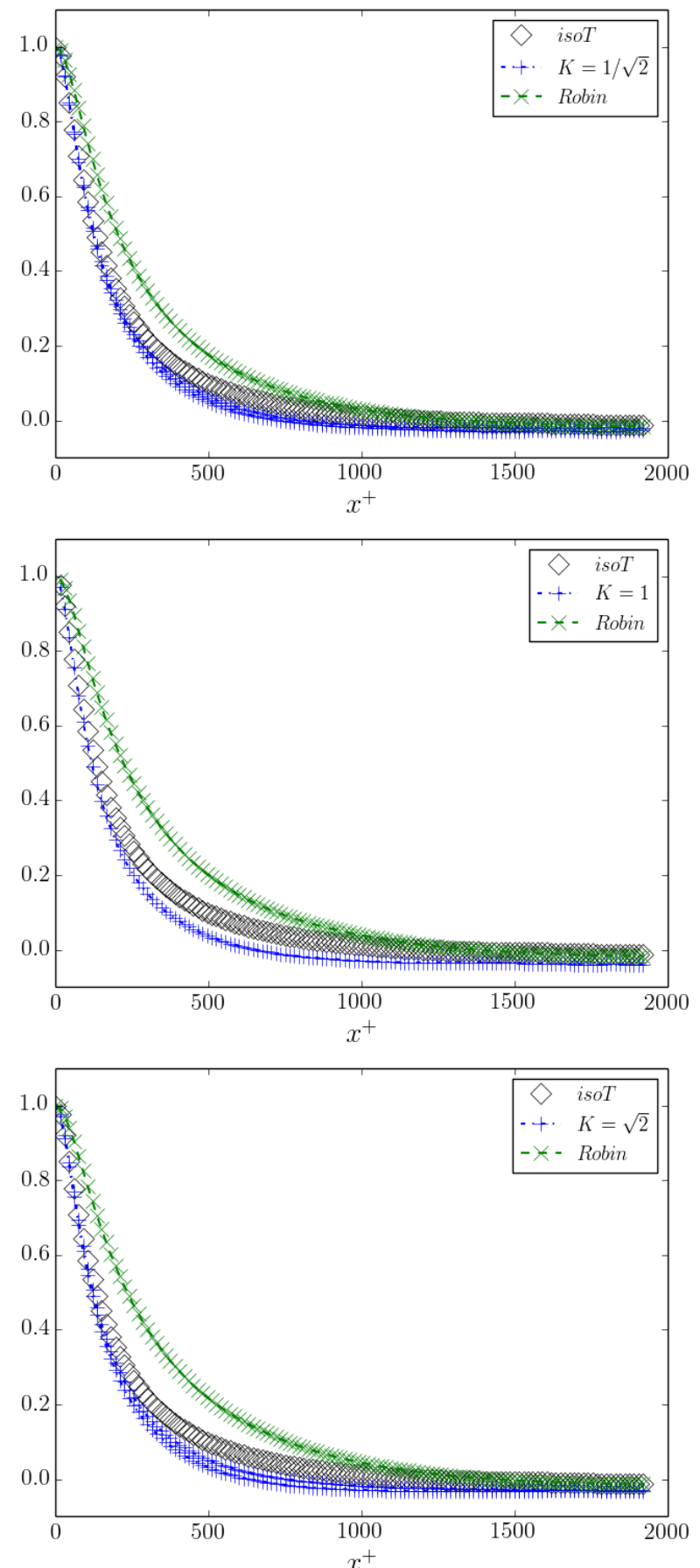

Figure 4: Two-point streamwise autocorrelation at the wall. Left: Temperature. Right: Wall-normal heat flux. Top: $K=1 / \sqrt{2}$. Middle: $K=1$. Bottom: $K=\sqrt{2}$. +: Conjugate. x: Robin. 
Two-point spanwise autocorrelation

(temperature)
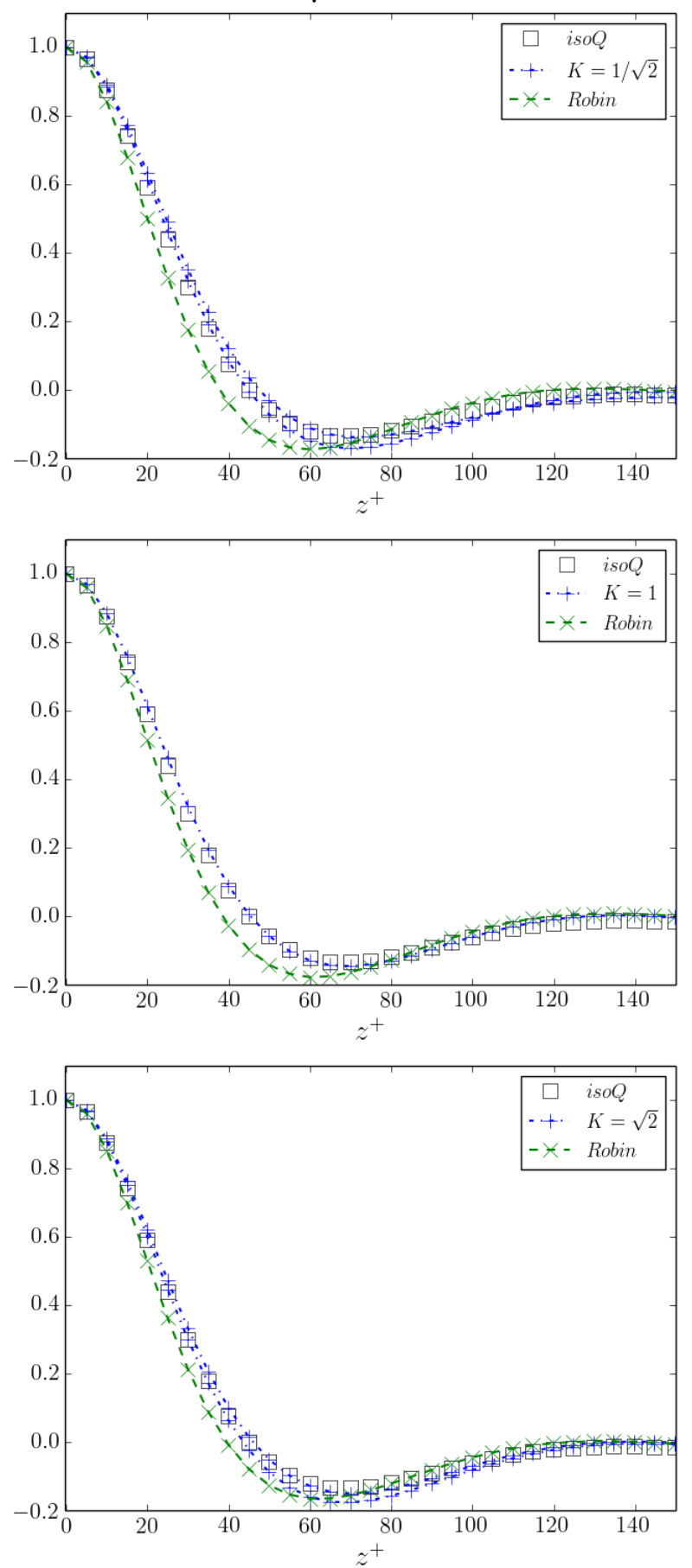

Two-point spanwise autocorrelation (wall-normal heat flux)
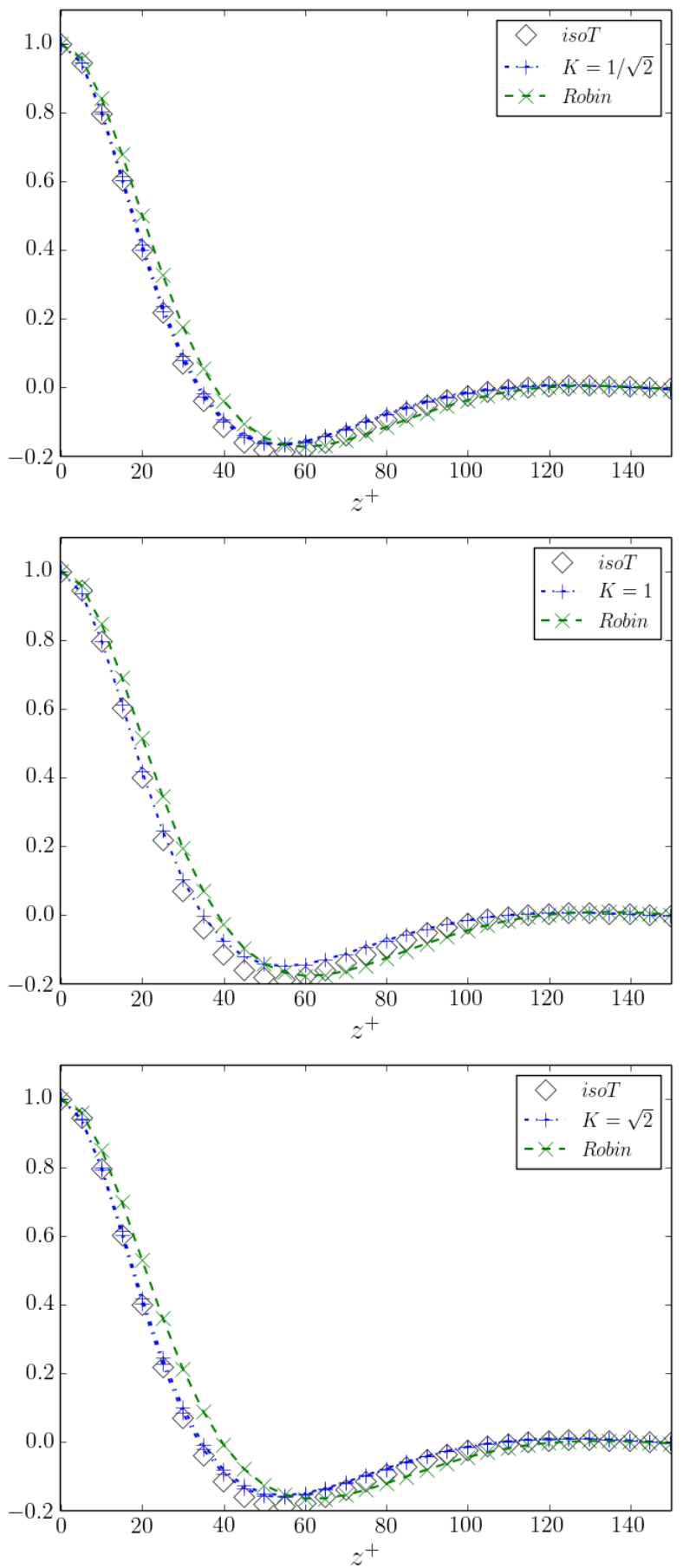

Figure 5: Two-point spanwise autocorrelation at the wall. Left: Temperature. Right: Wall-normal heat flux. Top: $K=1 / \sqrt{2}$. Middle: $K=1$. Bottom: $K=\sqrt{2}$. +: Conjugate. x: Robin. 

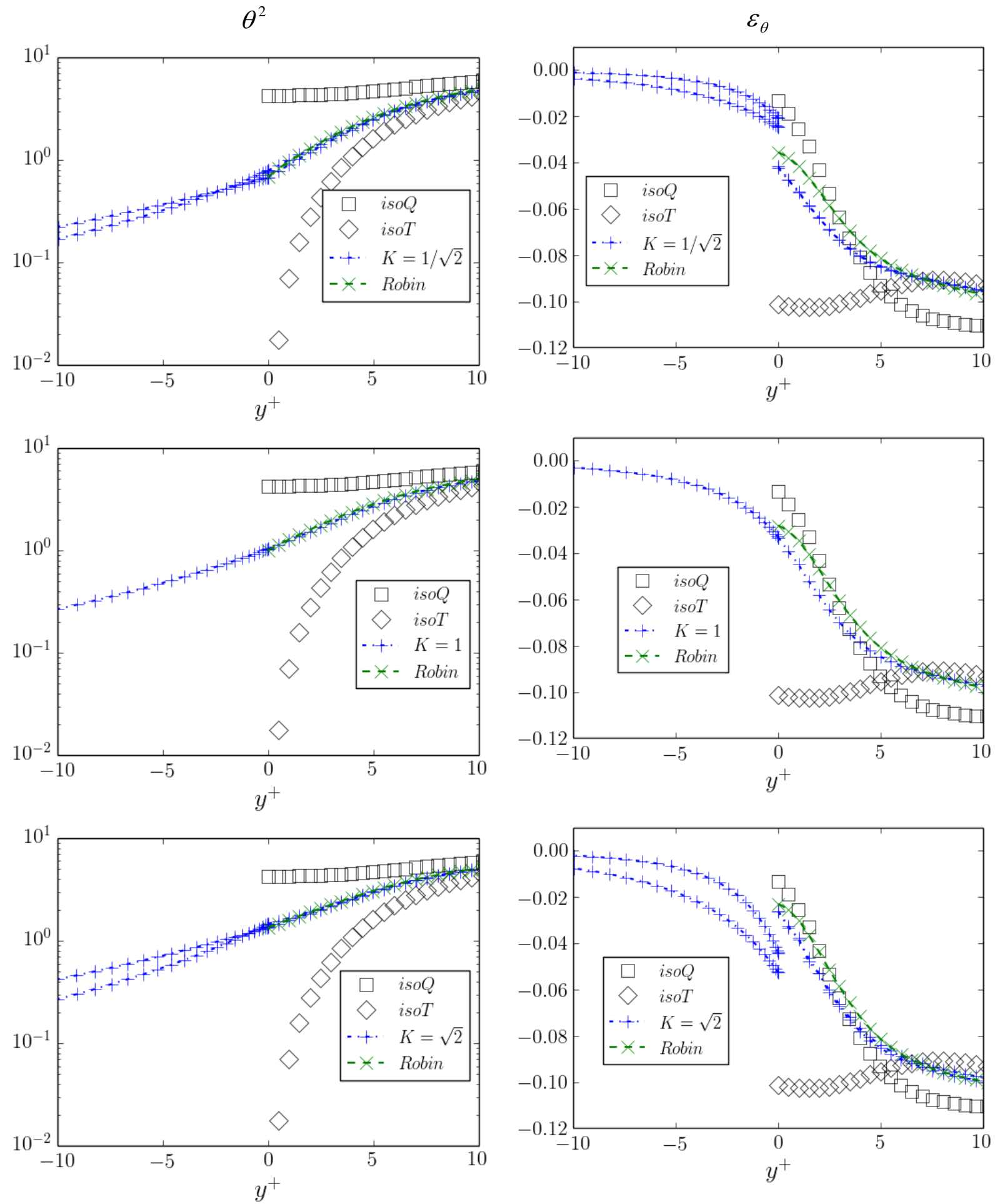

Figure 6: Left: Profile of $\theta^{2}$ around the fluid-solid interface. Right: Profile of $\varepsilon_{\theta}$ around the fluid-solid interface. 
Relative contribution of its wall-normal part to $\varepsilon_{\theta}$
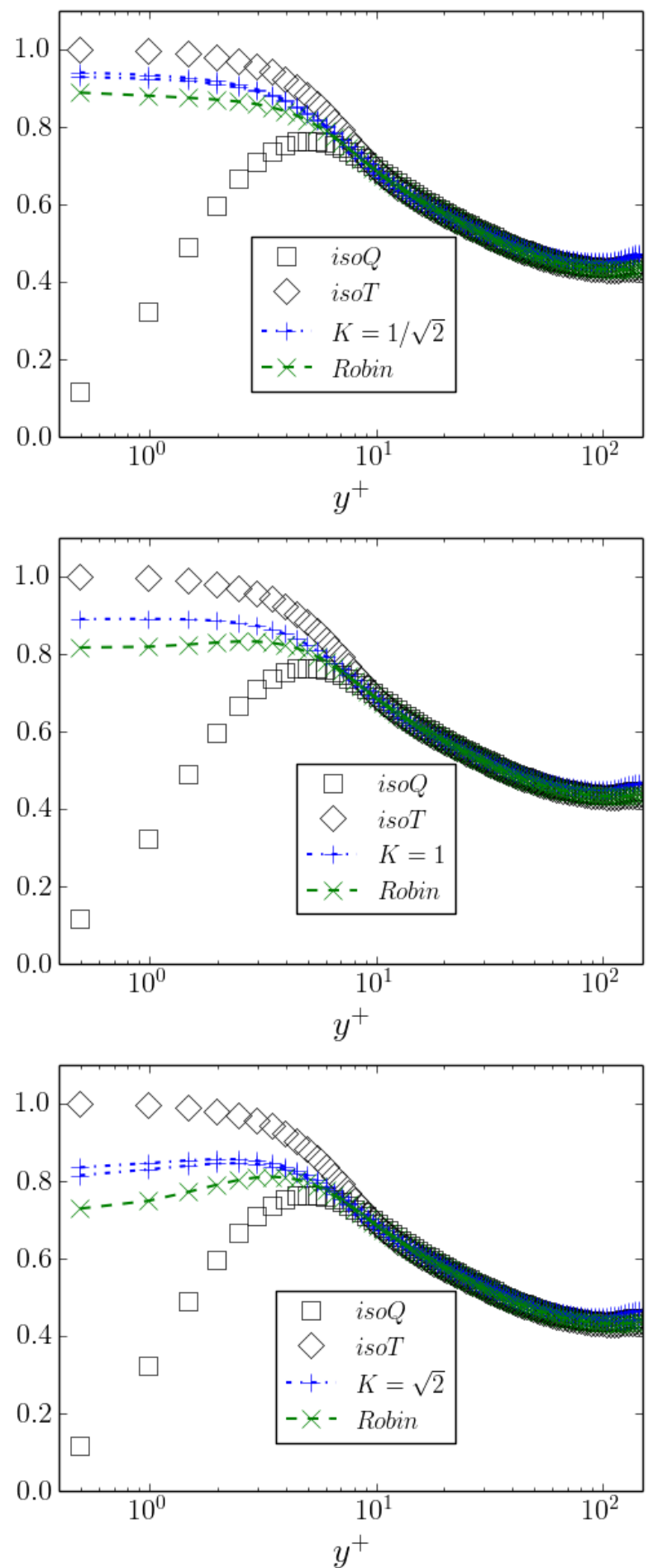

Scalings for $\theta^{2}$ and $\varepsilon_{\theta, f}$
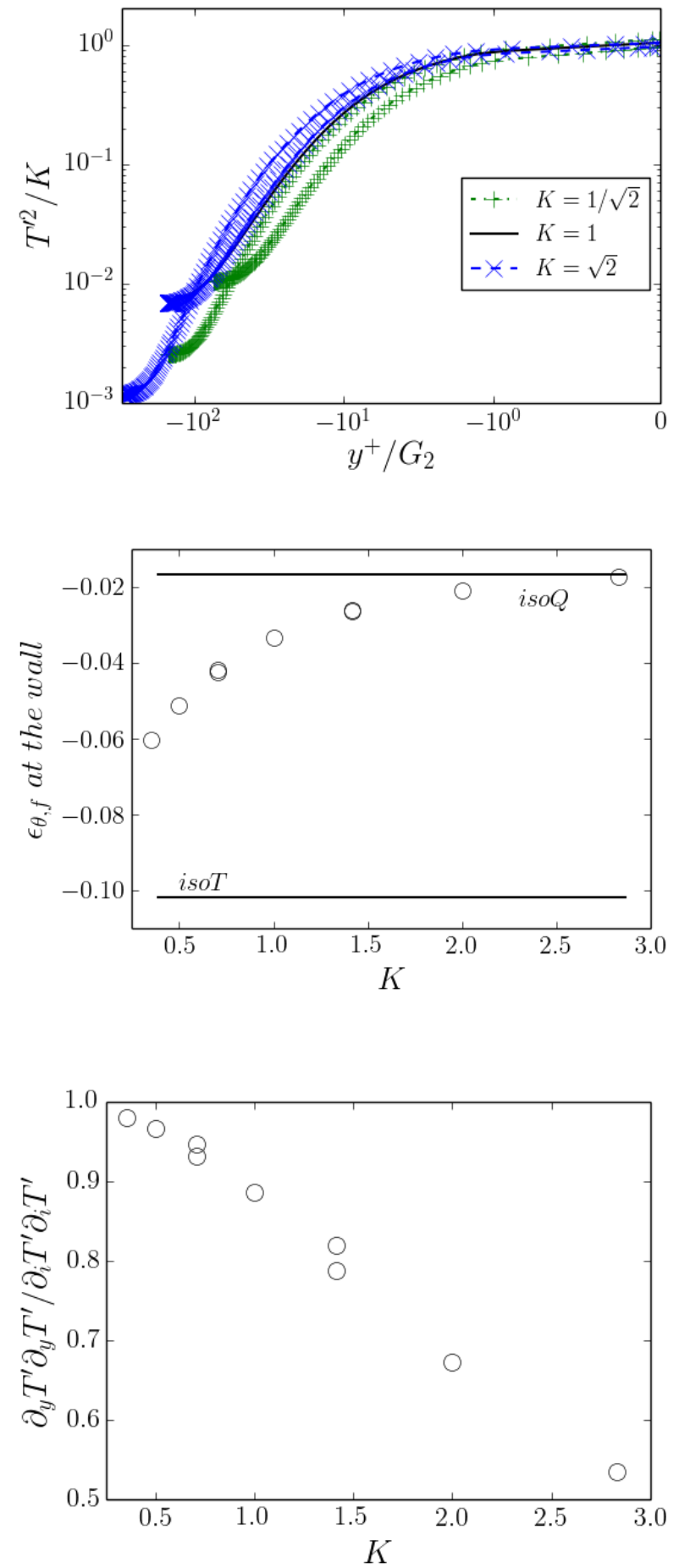

Figure 7: Left: Relative contribution to $\varepsilon_{\theta}$ of its wall-normal part. Right: Scaling for $\theta^{2}$ in the solid domain and for $\varepsilon_{\theta, f}$ and its wall normal contribution to at the fluid-solid interface. 